\title{
Controllability of Sobolev Type Fractional Evolution Systems
}

\author{
JinRong Wang, Michal Fečkan, and Yong Zhou \\ Communicated by Y. Charles Li, received September 27, 2012.
}

\begin{abstract}
The main purpose of this paper is to investigate a class of Sobolev type semilinear fractional evolution systems in a separable Banach space. Applying a suitable fixed point theorem as well as condensing mapping, controllability results for two class of control sets are established by means of the theory of propagation family and technique of measure of noncompactness. An application involving a partial differential equation with a Caupto fractional derivative is considered.
\end{abstract}

\section{CONTEnts}

1. Introduction $\quad 72$

2. Preliminaries $\quad 73$

3. Main results 76

4. Example $\quad 83$

$\begin{array}{ll}\text { References } & 85\end{array}$

1991 Mathematics Subject Classification. 47J35, 93B05, 93C25.

Key words and phrases. Sobolev type fractional evolution systems, propagation family, measure of noncompactness, mild solutions, controllability.

The first author's work was supported by the National Natural Science Foundation of China (11201091), Key Projects of Science and Technology Research in the Chinese Ministry of Education (211169), Key Support Subject (Applied Mathematics), Key Project on the Reforms of Teaching Contents and Course System of Guizhou Normal College and Doctor Project of Guizhou Normal College (13BS010); the second author acknowledges the support by Grants VEGA-MS 1/0507/11, VEGA-SAV 2/7140/27 and APVV-0134-10 and the third author acknowledges the support by National Natural Science Foundation of China (11271309), Specialized Research Fund for the Doctoral Program of Higher Education (20114301110001) and Key Projects of Hunan Provincial Natural Science Foundation of China (12JJ2001). 


\section{Introduction}

In this paper, we consider the following Sobolev type fractional evolution system in a separable Banach space $X$ :

$$
\left\{\begin{array}{l}
{ }_{0}^{C} D_{t}^{q}(E x(t))=A x(t)+E f(t, x(t))+E B u(t), t \in J:=[0, a], \\
E x(0)=E x_{0}, x_{0} \in D(E),
\end{array}\right.
$$

where ${ }_{0}^{C} D_{t}^{q}$ is the Caputo fractional derivative of order $0<q<1$ with the lower limit zero (see Definition 1.3), $A: D(A) \subset X \rightarrow X$ and $E: D(E) \subset X \rightarrow X$ are two closed linear operators and the pair $(A, B)$ generates an exponentially bounded propagation family $\{W(t), t \geq 0\}$ of $D(E)$ to $X$ (see Definition 2.11, Liang and Xiao [1] ). The state $x(\cdot)$ takes values in $X$ and the control function $u(\cdot)$ is given in $\mathscr{U}$, the Banach space of admissible control functions, where

$$
\mathscr{U}:=\left\{\begin{array}{l}
L^{p}(J, U), \text { for } q \in\left(\frac{1}{p}, 1\right) \text { with } 1<p<\infty, \\
L^{\infty}(J, U), \text { for } q \in(0,1),
\end{array}\right.
$$

and $U$ is a Banach space. $B$ is a bounded linear operator from $U$ into $D(E)$ and $f: J \times X \rightarrow D(E) \subset X$ will be specified later.

Sobolev type evolution equations often arise in various applications such as in the flow of fluid through fissured rocks, thermodynamics and shear in second order fluids. Meanwhile, fractional calculus was planted over three hundred years ago and provided an excellent tool for the description of memory and hereditary properties of various materials and processes. In particular, the subject of fractional differential equations is gaining much importance and attention. So-called fractional differential equations are specified by generalizing the standard integer order derivative to arbitrary order. Due to the effective memory function of fractional derivative, fractional differential equations have been widely used to describe many physical phenomena such as seepage flow in porous media and in fluid dynamic traffic model. For more interesting theory results and scientific applications of fractional differential equations, we cite the monographs $[\mathbf{2}, \mathbf{3}, \mathbf{4}, \mathbf{5}, \mathbf{6}]$, the research papers $[7,8,9,10,11,12,13,14,15,16,17,18,19,20,21,22,23,24,25$, $\mathbf{2 6}, \mathbf{2 7}, \mathbf{2 8}, \mathbf{2 9}, \mathbf{3 0}, \mathbf{3 1}]$ and the references therein.

In the past decade, many researchers have studied the existence and controllability of the mild solutions for Cauchy problem of all kinds of Sobolev type evolution equations under the various of conditions on the pair $(A, E)$. After reviewing these interesting results, the reader can find that $D(A) \subset D(E)$, boundedness or compactness of $E^{-1}$ are posed (see $[\mathbf{1 0}, \mathbf{3 2}, \mathbf{3 3}]$ ). In particular, Li et al. [34] obtained new existence results for Sobolev type fractional evolution equations by virtue of the theory of propagation family which generated by the pair $(A, E)$ via the techniques of the measure of noncompactness and the condensing maps. The restrict conditions on the $D(A), D(E)$ and $E^{-1}$ are removed.

However, to the best of our knowledge, controllability results of Sobolev type fractional evolution systems via the theory of propagation family have not been explored. Thus, we offer to study the controllability of the system (1) via the theory of propagation family $\{W(t), t \geq 0\}$ generating by the pair $(A, E)$. Our aim in this paper is to present sufficient conditions for the controllability results corresponding to two class of the possible admissible control sets. To simplify the process, we construct $\left\{\mathscr{T}_{(A, E)}(t), t \geq 0\right\}$ and $\left\{\mathscr{S}_{(A, E)}(t), t \geq 0\right\}$ associated with the pair $(A, E)$ and give their boundedness and norm continuity in the sense of uniform 
operator topology. Here, we mixed and modify the conditions and techniques used in $[\mathbf{2 6}, \mathbf{3 2}, \mathbf{3 4}, \mathbf{3 5}]$ to prove the controllability results.

To end this section, we recall the following known definitions of fractional calculus (see, e.g., $[\mathbf{3}, \mathbf{4}, \mathbf{5}]$ ).

DEFINITION 1.1. The fractional integral of order $\gamma$ with the lower limit zero for a function $f$ is defined as

$$
{ }_{0} I_{t}^{\gamma} f(t)=\frac{1}{\Gamma(\gamma)} \int_{0}^{t} \frac{f(s)}{(t-s)^{1-\gamma}} d s, t>0, \gamma>0,
$$

provided the right side is point-wise defined on $[0, \infty)$, where $\Gamma(\cdot)$ is the gamma function.

Definition 1.2. The Riemann-Liouville derivative of order $\gamma$ with the lower limit zero for a function $f:[0, \infty) \rightarrow \mathbb{R}$ can be written as

$$
{ }_{0}^{L} D_{t}^{\gamma} f(t)=\frac{1}{\Gamma(n-\gamma)} \frac{d^{n}}{d t^{n}} \int_{0}^{t} \frac{f(s)}{(t-s)^{\gamma+1-n}} d s, t>0, n-1<\gamma<n .
$$

DeFinition 1.3. The Caputo derivative of order $\gamma$ for a function $f:[0, \infty) \rightarrow \mathbb{R}$ can be written as

$$
{ }_{0}^{C} D_{t}^{\gamma} f(t)={ }_{0}^{L} D_{t}^{\gamma}\left[f(t)-\sum_{k=0}^{n-1} \frac{t^{k}}{k !} f^{(k)}(0)\right], t>0, n-1<\gamma<n .
$$

REMARK 1.4. (i) If $f(t) \in C^{1}[0, \infty)$, then ${ }_{0}^{C} D_{t}^{\gamma} f(t)={ }_{0} I_{t}^{1-\gamma} f^{\prime}(t), t>0$, $0<\gamma<1$. (ii) The Caputo derivative of a constant is equal to zero. (iii) If $f$ is an abstract function with values in $X$, then integrals which appear in Definitions 1.1 and 1.2 are taken in Bochner's sense.

\section{Preliminaries}

Let's recall some definitions and properties of measure of noncompactness and condensing maps (see, e.g., $[\mathbf{3 6}, \mathbf{3 7}, \mathbf{3 8}]$ ).

Definition 2.1. Let $Y^{+}$be the positive cone of an order Banach space $(Y, \leq)$. A function $\Phi$ defined on the set of all bounded subsets of the Banach space $X$ with values in $Y^{+}$is called a measure of noncompactness (MNC) on $X$ if $\Phi(\overline{c o} \Omega)=\Phi(\Omega)$ for all bounded subsets $\Omega \subset X$, where $\overline{c o} \Omega$ stands for the closed convex hull of $\Omega$.

The $M N C \Phi$ is said to be:

(i) Monotone: if for all bounded subsets $\Omega_{1}, \Omega_{2}$ of $X, \Omega_{1} \subseteq \Omega_{2}$ implies $\Phi\left(\Omega_{1}\right) \leq$ $\Phi\left(\Omega_{2}\right)$;

(ii) Nonsingular: if $\Phi(\{\theta\} \cup \Omega)=\Phi(\Omega)$ for every $\theta \in X$ and every nonempty subset $\Omega \subseteq X$;

(iii) Regular: if $\Phi(\Omega)=0$ if and only if $\Omega$ is relatively compact in $X$.

One of the most important examples of $M N C$ is the noncompactness measure of Hausdorff $\chi$ defined on each bounded subset $\Omega$ of $X$ by

$$
\chi(\Omega)=\inf \{\varepsilon>0: \Omega \text { has a finite } \varepsilon \text {-net in } X\} .
$$

It is well known that Hausdorff $M N C \chi$ enjoys the above properties (i)-(iii) and other properties (see $[\mathbf{3 7}, \mathbf{3 8}]$ ).

(iv) $\chi\left(\Omega_{1}+\Omega_{2}\right) \leq \chi\left(\Omega_{1}\right)+\chi\left(\Omega_{2}\right)$, where $\Omega_{1}+\Omega_{2}=\left\{x+y: x \in \Omega_{1}, y \in \Omega_{2}\right\}$;

(v) $\chi\left(\Omega_{1} \cup \Omega_{2}\right) \leq \max \left\{\chi\left(\Omega_{1}\right), \chi\left(\Omega_{2}\right)\right\}$; 
(vi) $\chi(\lambda \Omega) \leq|\lambda| \chi(\Omega)$ for any $\lambda \in \mathbb{R}$

(vii) If the map $Q: D(Q) \subseteq X \rightarrow Z$ is Lipschitz continuous with constant $k$, then $\chi_{Z}(Q \Omega) \leq k \chi(\Omega)$ for any bounded subset $\Omega \subseteq D(\Omega)$, where $Z$ is a Banach space.

Now, let $G: J \rightarrow 2^{X}$ be a multifunction where $2^{X}$ denotes the class of all nonempty subsets of $X$. It is called:

(i) Integrable: if it admits a Bochner integrable selection $g: J \rightarrow X, g(t) \in G(t)$ for a.e. $t \in J$.

(ii) Integrably bounded: if there exists a function $\kappa(\cdot) \in L^{1}\left(J, \mathbb{R}^{+}\right)$such that $\|G(t)\|:=\sup \{\|g\|: g \in G(t)\} \leq \kappa(t)$ for a.e. $t \in J$.

Lemma 2.2. (see Theorem 4.2.3, [38]) For an integrable, integrably bounded multifunction $G: J \rightarrow 2^{X}$ where $X$ is a separable Banach space, let $\chi(G(t)) \leq g(t)$ for a.e. $t \in J$, where $g \in L^{1}\left(J, \mathbb{R}^{+}\right)$. Then $\chi\left(\int_{0}^{t} G(s) d s\right) \leq \int_{0}^{t} g(s) d s$ for all $t \in J$.

We also recall definition of condensing maps and fixed point theorems via condensing maps (see, e.g., $[\mathbf{3 6}, \mathbf{3 8}]$ ).

Definition 2.3. Let $\beta$ be a monotone nonsingular MNC in Banach space $Y$. A continuous map $\mathscr{P}: Y \subseteq Y \rightarrow Y$ is called condensing with respect to a $M N C$ $\beta$ (or $\beta$-condensing) if for every bounded set $\Omega \subseteq Y$ which which is not relatively compact, we have $\beta(\mathscr{P}(\Omega)) \nsupseteq \beta(\Omega)$.

THEOREM 2.4. Let $\mathfrak{B}$ be a bounded convex closed subset of $Y$ and $\mathscr{P}: \mathfrak{B} \rightarrow \mathfrak{B}$ a $\beta$-condensing map. Then Fix $\mathscr{P}=\{x: x=\mathscr{P}(x)\}$ is nonempty.

THEOREM 2.5. Let $\mathcal{V} \subset Y$ be a bounded open neighborhood of zero and $\mathscr{H}$ : $\overline{\mathcal{V}} \rightarrow Y$ a $\beta$-condensing map satisfying the boundary condition $x \neq \hat{\lambda} \mathscr{H}(x)$ for all $x \in \partial V$ and $\hat{\lambda} \in(0,1]$. Then Fix $\mathscr{H}=\{x: x=\mathscr{H}(x)\}$ is a nonempty compact set.

Next, we recall the concept of exponentially bounded propagation family (see Definition 1.4, [1] ).

DEFINITION 2.6. A strongly continuous operator family $\{W(t), t \geq 0\}$ of $D(E)$ to a Banach space $X$ satisfying that $\{W(t), t \geq 0\}$ is exponentially bounded, which means that there exist $\omega>0$ and $M>0$ such that $\|W(t) x\| \leq M e^{\omega t}\|x\|$ for any $x \in D(E)$ and $t \geq 0$, is called an exponentially bounded propagation family for the following abstract degenerate Cauchy problem

$$
\left\{\begin{array}{l}
(E x(t))^{\prime}=A x(t), t \in J, \\
E x(0)=E x_{0}, x_{0} \in D(E),
\end{array}\right.
$$

if for $\lambda>\omega$,

$$
(\lambda E-A)^{-1} E x=\int_{0}^{\infty} e^{-\lambda t} W(t) x d t, x \in D(E) .
$$

In this case, we say that the problem (2) has an exponentially bounded propagation family $\{W(t), t \geq 0\}$.

Moreover, if (3) holds, we also say that the pair $(A, E)$ generates an exponentially bounded propagation family $\{W(t), t \geq 0\}$.

REMARK 2.7. Since $D(E) \subset X$ is dense, $W(t)$ can be uniquely extended on $X$ as a linear bounded mapping so that $\|W(t) x\| \leq M e^{\omega t}\|x\|$ for any $x \in X$ and $t \geq 0$. From now on, we consider such $W(t)$ on $X$ directly. 
Denote

(4) $\mathscr{T}_{(A, E)}(t)=\int_{0}^{\infty} \xi_{q}(\theta) W\left(t^{q} \theta\right) d \theta, \mathscr{S}_{(A, E)}(t)=q \int_{0}^{\infty} \theta \xi_{q}(\theta) W\left(t^{q} \theta\right) d \theta$, where

$$
\begin{aligned}
\xi_{q}(\theta) & =\frac{1}{q} \theta^{-\left(1+\frac{1}{q}\right)} \varpi_{q}\left(\theta^{-\frac{1}{q}}\right) \geq 0 \\
\varpi_{q}(\theta) & =\frac{1}{\pi} \sum_{n=1}^{\infty}(-1)^{n-1} \theta^{-q n-1} \frac{\Gamma(n q+1)}{n !} \sin (n \pi q) .
\end{aligned}
$$

And, $\xi_{q}$ is a probability density function defined on $(0, \infty)$, that is

$(6) \xi_{q}(\theta) \geq 0, \theta \in(0, \infty), \int_{0}^{\infty} \xi_{q}(\theta) d \theta=1$, and $\int_{0}^{\infty} \theta \xi_{q}(\theta) d \theta=\frac{1}{\Gamma(1+q)}$.

Using the similar method in $[\mathbf{1 2}, \mathbf{1 3}, \mathbf{3 4}]$, we can introduce the following definition of mild solution for the system (1).

Definition 2.8. For each $u \in \mathscr{U}$ and $x_{0} \in D(E)$, a mild solution of the system (1) we mean a function $x \in C(J, X)$ which satisfies

$$
\begin{aligned}
x(t)= & \mathscr{T}_{(A, E)}(t) x_{0}+\int_{0}^{t}(t-s)^{q-1} \mathscr{S}_{(A, E)}(t-s) f(s, x(s)) d s \\
& +\int_{0}^{t}(t-s)^{q-1} \mathscr{S}_{(A, E)}(t-s) B u(s) d s, t \in J .
\end{aligned}
$$
paper.

The following results of $\mathscr{T}_{(A, E)}(\cdot)$ and $\mathscr{S}_{(A, E)}(\cdot)$ will be used throughout this

Lemma 2.9. Suppose the pair $(A, E)$ generates an exponentially bounded propagation family $\{W(t), t \geq 0\}$. If $\{W(t), t \geq 0\}$ is a norm continuous family for $t>0$ and $\|W(t)\| \leq M_{1}$ for $t \geq 0$, then the following two properties hold:

(i) For any fixed $t \geq 0, \mathscr{T}_{(A, E)}(t)$ and $\mathscr{S}_{(A, E)}(t)$ are bounded operators on $X$, i.e., for any $x \in X$,

$$
\left\|\mathscr{T}_{(A, E)}(t) x\right\| \leq M_{1}\|x\| \text { and }\left\|\mathscr{S}_{(A, E)}(t) x\right\| \leq \frac{M_{1}}{\Gamma(q)}\|x\| .
$$

(ii) $\left\{\mathscr{T}_{(A, E)}(t), t \geq 0\right\}$ and $\left\{\mathscr{S}_{(A, E)}(t), t \geq 0\right\}$ are norm continuous family for $t>0$ in the sense of uniform operator topology.

Proof. The first assertion has been proved (see Remark 2.1.3, [34]). Next, we verify the second assertion. We only need to prove that $\left\|\mathscr{T}_{(A, E)}\left(t_{1}\right)-\mathscr{T}_{(A, E)}\left(t_{2}\right)\right\|$ and $\left\|\mathscr{S}_{(A, E)}\left(t_{1}\right)-\mathscr{S}_{(A, E)}\left(t_{2}\right)\right\|$ tend to zero as $t_{1} \rightarrow t_{2}$ respectively in the sense of uniform operator topology.

For $0<t_{1}<t_{2}<\infty$, a simple computation implies

$$
\begin{gathered}
\left\|\mathscr{T}_{(A, E)}\left(t_{1}\right)-\mathscr{T}_{(A, E)}\left(t_{2}\right)\right\| \leq \int_{0}^{\infty} \xi_{q}(\theta)\left\|W\left(t_{1}^{q} \theta\right)-W\left(t_{2}^{q} \theta\right)\right\| d \theta, \\
\left\|\mathscr{S}_{(A, E)}\left(t_{1}\right)-\mathscr{S}_{(A, E)}\left(t_{2}\right)\right\| \leq q \int_{0}^{\infty} \theta \xi_{q}(\theta)\left\|W\left(t_{1}^{q} \theta\right)-W\left(t_{2}^{q} \theta\right)\right\| d \theta .
\end{gathered}
$$

Note that $\left\|W\left(t_{1}^{q} \theta\right)-W\left(t_{2}^{q} \theta\right)\right\| \rightarrow 0$ as $t_{1} \rightarrow t_{2}$ in the sense of uniform operator topology for any fixed $\theta>0$. Linking (6) and (7), (8), one can obtain the second assertion immediately. The proof is complete. 


\section{Main results}

In this section, we study the controllability of the system (1) by utilizing the theory of propagation family and techniques of measure of noncompactness.

Definition 3.1. The system (1) is said to be controllable on the interval $J$ if for every $x_{0} \in D(E)$ and every $x_{1} \in D(E)$ there exists a control $u \in \mathscr{U}$ such that the mild solution $x$ of system (1) satisfies $x(a)=x_{1}$.

We pose the following assumptions:

$\left[H_{1}\right]$ : The pair $(A, B)$ generates an exponentially bounded propagation family $\{W(t), t \geq 0\}$ of $D(E)$ to $X$. $t \geq 0$.

$\left[H_{2}\right]:\{W(t), t \geq 0\}$ is norm continuous family for $t>0$ and $\|W(t)\| \leq M_{1}$ for

$\left[H_{3}\right]$ : The control function $u(\cdot)$ takes from $\mathscr{U}$, the Banach space of admissible control functions, either $\mathscr{U}:=L^{p}(J, U)$ for $q \in\left(\frac{1}{p}, 1\right)$ with $1<p<\infty$ or $\mathscr{U}:=$ $L^{\infty}(J, U)$ for $q \in(0,1)$ where $U$ is a Banach space.

$\left[H_{4}\right]: B: U \rightarrow D(E)$ is a bounded linear operator and a linear operator $\mathbb{W}: \mathscr{U} \rightarrow X$ defined by

$$
\mathbb{W} u=\int_{0}^{a}(a-s)^{q-1} \mathscr{S}_{(A, E)}(a-s) B u(s) d s
$$

has a bounded right inverse operator $\mathbb{W}^{-1}: X \rightarrow \mathscr{U}$.

It is easy to see that $\mathbb{W} u \in X$ and $\mathbb{W}$ is well defined due to the following fact:

$$
\begin{aligned}
\|\mathbb{W} u\| & =\left\|\int_{0}^{a}(a-s)^{q-1} \mathscr{S}_{(A, E)}(a-s) B u(s) d s\right\| \\
\leq & \frac{M_{1}\|B\|}{\Gamma(q)} \int_{0}^{a}(a-s)^{q-1}\|u(s)\| d s \\
& \leq\left\{\begin{array}{c}
\frac{M_{1}\|B\|}{\Gamma(q)}\left(\frac{p-1}{q p-1} a^{\frac{q p-1}{p-1}}\right)^{\frac{p-1}{p}}\|u\|_{\mathscr{U}}, \text { if } q \in\left(\frac{1}{p}, 1\right), \\
u \in \mathscr{U}=L^{p}(J, U), 1<p<\infty \\
\frac{M_{1}\|B\| a^{q}}{\Gamma(q+1)}\|u\|_{\mathscr{U}}, \text { if } q \in(0,1), u \in \mathscr{U}=L^{\infty}(J, U) .
\end{array}\right.
\end{aligned}
$$

Meanwhile,

$$
\int_{0}^{t}(t-s)^{q-1}\|u(s)\| d s \leq K_{q}\|u\|_{\mathscr{U}}
$$

where

$K_{q}:=\left\{\begin{array}{l}\left(\frac{p-1}{q p-1} a^{\frac{q p-1}{p-1}}\right)^{\frac{p-1}{p}}\|u\|_{\mathscr{U}}, \text { if } q \in\left(\frac{1}{p}, 1\right), u \in \mathscr{U}=L^{p}(J, U), 1<p<\infty, \\ \frac{a^{q}}{q}\|u\|_{\mathscr{U}}, \text { if } q \in(0,1), u \in \mathscr{U}=L^{\infty}(J, U),\end{array}\right.$

for any $t \in J$.

Next we assume:

$\left[H_{5}\right]: f$ satisfies the following two conditions:

(i) For each $x \in X$ the function $f(\cdot, x): J \rightarrow D(E) \subset X$ is strongly measurable and for each $t \in J$, the function $f(t, \cdot): X \rightarrow D(E) \subset X$ is continuous.

(ii) For each $k>0$, there is a measurable function $g_{k}$ such that

$$
\sup _{\|x\| \leq k}\|f(t, x)\| \leq g_{k}(t), \text { with }\left\|g_{k}\right\|_{\infty}:=\sup _{s \in J} g_{k}(s)<\infty
$$




$$
\sup _{t \in J} \int_{0}^{t}(t-s)^{q-1} g_{k}(s) d s \leq \gamma k,
$$

for any $k>0$ sufficiently large and some $\gamma$.

(iii) There exists a positive constant $L>0$ such that

$$
\chi(f(t, D)) \leq L \chi(D)
$$

for any bounded set $D \subset X$ and a.e. $t \in J$.

The first step in studying the controllability problem is to determine if an objective can be reached by some suitable control. A standard approach is to transform the controllability problem into a fixed point problem for an appropriate operator in a function space. For the sake of simplicity, we present the standard framework to deal with controllability problems here.

Based on our assumptions, for an arbitrary function $x(\cdot)$, it is suitable to define the following control formula

$$
\text { (10) } u(t)=\mathbb{W}^{-1}\left[x_{1}-\mathscr{T}_{(A, E)}(a) x_{0}-\int_{0}^{a}(a-s)^{q-1} \mathscr{S}_{(A, E)}(a-s) f(s, x(s)) d s\right] \text {. }
$$

In what follows, it is necessary to show that when using the control $u$ in (10), the operator $\mathcal{P}$ defined by

$$
\begin{aligned}
(\mathcal{P} x)(t)= & \mathscr{T}_{(A, E)}(t) x_{0}+\int_{0}^{t}(t-s)^{q-1} \mathscr{S}_{(A, E)}(t-s) f(s, x(s)) d s \\
& +\int_{0}^{t}(t-s)^{q-1} \mathscr{S}_{(A, E)}(t-s) B u(s) d s, \text { for } t \in J,
\end{aligned}
$$

from $C(J, X)$ into $C(J, X)$, has a fixed point. Clearly, this fixed point is just a mild solution of system (1). Further, one can check

$$
\begin{aligned}
(\mathcal{P} x)(a)= & \mathscr{T}_{(A, E)}(a) x_{0}+\int_{0}^{a}(a-s)^{q-1} \mathscr{S}_{(A, E)}(a-s) f(s, x(s)) d s \\
& +\int_{0}^{a}(a-s)^{q-1} \mathscr{S}_{(A, E)}(a-s) \\
& \times B \mathbb{W}^{-1}\left[x_{1}-\mathscr{T}_{(A, E)}(a) x_{0}\right. \\
& \left.-\int_{0}^{a}(a-\tau)^{q-1} \mathscr{S}_{(A, E)}(a-\tau) f(\tau, x(\tau)) d \tau\right] d s \\
= & x_{1},
\end{aligned}
$$

which means that $u$ steers the fractional system (1) from $x_{0}$ to $x_{1}$ in finite time $a$. Consequently, we can claim the system (1) is controllable on $J$.

For each number $k>0$, define

$$
\mathcal{B}_{k}=\{x \in C(J, X):\|x(t)\| \leq k, t \in J\} .
$$

Of course, $\mathcal{B}_{k}$ is clearly a bounded, closed, convex subset in $C(J, X)$.

Under the assumptions $\left[H_{1}\right]-\left[H_{5}\right]$, we will establish some important results as follows.

Lemma 3.2. Assuming

$$
\rho:=\left\{\begin{array}{l}
\frac{\gamma M_{1}}{\Gamma(q)}\left(1+\frac{\sqrt{a} M_{1}\|B\| K_{q}\left\|\mathbb{W}^{-1}\right\|}{\Gamma(q)}\right)<1, \text { if } \mathscr{U}=L^{2}(J, U), \\
\frac{\gamma M_{1}}{\Gamma(q)}\left(1+\frac{M_{1}\|B\| K_{q}\left\|\mathbb{W}^{-1}\right\|}{\Gamma(q)}\right)<1, \text { if } \mathscr{U}=L^{\infty}(J, U),
\end{array}\right.
$$


there exists a constant $K \geq \frac{M^{*}}{1-\rho}$ such that $\mathcal{P} \mathcal{B}_{K} \subset \mathcal{B}_{K}$, where

$$
M^{*}:=\left\{\begin{array}{l}
M_{1}\left\|x_{0}\right\|+\frac{\sqrt{a} M_{1}\|B\|}{\Gamma(q)} K_{q}\left\|\mathbb{W}^{-1}\right\|\left(\left\|x_{1}\right\|+M_{1}\left\|x_{0}\right\|\right), \text { if } \mathscr{U}=L^{2}(J, U), \\
M_{1}\left\|x_{0}\right\|+\frac{M_{1}\|B\|}{\Gamma(q)} K_{q}\left\|\mathbb{W}^{-1}\right\|\left(\left\|x_{1}\right\|+M_{1}\left\|x_{0}\right\|\right), \text { if } \mathscr{U}=L^{\infty}(J, U) .
\end{array}\right.
$$

Proof. Let $x \in \mathcal{B}_{K}$. For $t \in J$, using our assumptions and Lemma 2.9(i), we obtain

$$
\begin{aligned}
\|(\mathcal{P} x)(t)\| \leq & M_{1}\left\|x_{0}\right\|+\frac{M_{1}}{\Gamma(q)} \int_{0}^{t}(t-s)^{q-1} g_{K}(s) d s+ \\
& \frac{M_{1}\|B\|}{\Gamma(q)} \int_{0}^{t}(t-s)^{q-1}\|u(s)\| d s \\
\leq & M_{1}\left\|x_{0}\right\|+\frac{M_{1} \gamma K}{\Gamma(q)}+\frac{M_{1}\|B\|}{\Gamma(q)} K_{q}\|u\|_{\mathscr{U}} \\
= & \rho K+M^{*} \\
\leq & K
\end{aligned}
$$

where we note that the control $u$ defined in (10) satisfies

$$
\begin{aligned}
\|u(t)\| & \leq\left\|\mathbb{W}^{-1}\right\|\left\|x_{1}-\mathscr{T}_{(A, E)}(a) x_{0}-\int_{0}^{a}(a-s)^{q-1} \mathscr{S}_{(A, E)}(a-s) f(s, x(s)) d s\right\| \\
& \leq\left\|\mathbb{W}^{-1}\right\|\left(\left\|x_{1}\right\|+M_{1}\left\|x_{0}\right\|+\frac{M_{1}}{\Gamma(q)} \gamma K\right),
\end{aligned}
$$

which implies that

$(13)\|u\|_{\mathscr{U}} \leq\left\{\begin{array}{l}\sqrt{a}\left\|\mathbb{W}^{-1}\right\|\left(\left\|x_{1}\right\|+M_{1}\left\|x_{0}\right\|+\frac{M_{1}}{\Gamma(q)} \gamma K\right), \text { if } \mathscr{U}=L^{2}(J, U), \\ \left\|\mathbb{W}^{-1}\right\|\left(\left\|x_{1}\right\|+M_{1}\left\|x_{0}\right\|+\frac{M_{1}}{\Gamma(q)} \gamma K\right), \text { if } \mathscr{U}=L^{\infty}(J, U) .\end{array}\right.$

Hence, $\mathcal{P} \mathcal{B}_{K} \subset \mathcal{B}_{K}$ for any $K \geq \frac{M^{*}}{1-\rho}$ sufficiently large. The proof is complete.

Lemma 3.3. The operator $\mathcal{P}$ defined by (11) is continuous.

Proof. Let $\left\{x_{m}\right\}_{m \in N} \subseteq \mathcal{B}_{K}$ be a sequence such that $x_{m} \rightarrow x$ as $m \rightarrow \infty$. Note that $(t-s)^{q-1} f\left(s, x_{m}(s)\right) \rightarrow(t-s)^{q-1} f(s, x(s))$ as $m \rightarrow \infty$ for very $t \in J$ and almost each $s \in[0, t]$ and

$$
(t-s)^{q-1}\left\|f\left(s, x_{m}(s)\right)-f(s, x(s))\right\| \leq 2(t-s)^{q-1} g_{K}(s) .
$$


Since $\int_{0}^{t}(t-s)^{q-1} g_{K}(s) \leq \frac{\left\|g_{K}\right\|_{\infty}}{q}$, by the Lebesgue's Dominated Convergence Theorem, we get

$$
\begin{aligned}
& \left\|\left(\mathcal{P} x_{m}\right)(t)-(\mathcal{P} x)(t)\right\| \\
\leq & \frac{M_{1}}{\Gamma(q)} \int_{0}^{t}(t-s)^{q-1}\left[\left\|f\left(s, x_{m}(s)\right)-f(s, x(s))\right\|\right. \\
+ & \left.\|B\|\left\|\mathbb{W}^{-1}\right\| \int_{0}^{a}(a-z)^{q-1}\left\|f\left(z, x_{m}(z)\right)-f(z, x(z))\right\| d z\right] d s \\
= & \frac{M_{1}}{\Gamma(q)} \int_{0}^{t}(t-s)^{q-1}\left\|f\left(s, x_{m}(s)\right)-f(s, x(s))\right\| d s \\
+ & \frac{M_{1}\|B\|\left\|\mathbb{W}^{-1}\right\| a^{q}}{\Gamma(q+1)}\left\|\int_{0}^{a}(a-s)^{q-1}\right\| f\left(s, x_{m}(s)\right)-f(z, x(s)) \| d s \\
\rightarrow & 0, \text { as } m \rightarrow \infty,
\end{aligned}
$$

for $t \in J$. This yields that $\mathcal{P}$ is continuous. The proof is complete.

Let $\chi$ be a Hausdorff $M N C$ in $X$. Consider the measure of noncompactness $\nu$ in the space $C(J, X)$ with values in the cone $\mathbb{R}^{2}$ of the way: for every bounded subset $\Omega \subset C(J, X)$,

$$
\nu:=\left(\psi(\Omega), \bmod _{c}(\Omega)\right)
$$

where $\psi(\Omega):=\sup _{t \in J} \chi(\Omega(t))$ and $\bmod _{c}(\Omega)=\lim _{\delta \rightarrow 0} \sup _{x \in \Omega} \max _{\left|t_{1}-t_{2}\right| \leq \delta} \| x\left(t_{1}\right)-$ $x\left(t_{2}\right) \|$.

Lemma 3.4. Assume

$$
\frac{a^{q} M_{1}}{\Gamma(q+1)} L\left[1+\frac{a^{q} M_{1}}{\Gamma(q+1)}\|B\|\left\|\mathbb{W}^{-1}\right\|\right]<1 .
$$

If $\nu\left(\mathcal{P}\left(\mathcal{B}_{K}\right)\right) \geq \nu\left(\mathcal{B}_{K}\right)$ then $\psi\left(\mathcal{B}_{K}\right)=0$. set

Proof. Clearly $\mathcal{B}_{K} \subset C(J, X)$ is nonempty and bounded. For any $t \in J$, we

$$
\Theta\left(\mathcal{B}_{K}(t)\right)=\int_{0}^{t} G(s) d s
$$

where a function $s \in[0, t] \multimap G(s)$ is defined as:

$$
\begin{aligned}
G(s)= & \left\{(t-s)^{q-1} \mathscr{S}_{(A, E)}(t-s) f(s, x(s))\right. \\
& \left.+(t-s)^{q-1} \mathscr{S}_{(A, E)}(t-s) B u(s): x \in \mathcal{B}_{K}\right\}
\end{aligned}
$$


and $u(t)$ is given by (10). It is obvious that $G$ is integrable and integrably bounded. Moreover, a simple computation implies that

$$
\begin{aligned}
\chi(G(s)) \leq & \frac{M_{1}}{\Gamma(q)}(t-s)^{q-1} \chi(\{f(s, x(s)) \\
& +B \mathbb{W}^{-1}\left[x_{1}-\mathscr{T}_{(A, E)}(a) x_{0}\right. \\
& \left.\left.\left.-\int_{0}^{a}(a-s)^{q-1} \mathscr{S}_{(A, E)}(a-s) f(s, x(s)) d s\right]: x \in \mathcal{B}_{K}\right\}\right) \\
\leq & \frac{M_{1}}{\Gamma(q)}(t-s)^{q-1}\left[\chi\left(\left\{f\left(s, \mathcal{B}_{K}(s)\right)\right\}\right)\right. \\
& +\chi\left(\left\{B \mathbb { W } ^ { - 1 } \left[x_{1}-\mathscr{T}_{(A, E)}(a) x_{0}\right.\right.\right. \\
& \left.\left.\left.\left.-\int_{0}^{a}(a-s)^{q-1} \mathscr{S}_{(A, E)}(a-s) f\left(s, \mathcal{B}_{K}(s)\right) d s\right]\right\}\right)\right] \\
\leq & \frac{M_{1}}{\Gamma(q)}(t-s)^{q-1}\left[L \chi\left(\mathcal{B}_{K}(s)\right)\right. \\
& \left.+\frac{M_{1}}{\Gamma(q)}\|B\|\left\|\mathbb{W}^{-1}\right\|\left(\int_{0}^{a}(a-s)^{q-1} L \chi\left(\mathcal{B}_{K}(s)\right) d s\right)\right] \\
\leq & \frac{M_{1}}{\Gamma(q)}(t-s)^{q-1} L\left[1+\frac{a^{q} M_{1}}{\Gamma(q+1)}\|B\|\left\|\mathbb{W}^{-1}\right\|\right] \psi\left(\mathcal{B}_{K}\right):=\kappa(s) .
\end{aligned}
$$

By Lemma 2.2, we have

$$
\begin{aligned}
\chi\left(\Theta\left(\mathcal{B}_{K}(t)\right)\right) & \leq \int_{0}^{t} \kappa(s) d s \\
& \leq \frac{t^{q} M_{1}}{\Gamma(q+1)} L\left[1+\frac{a^{q} M_{1}}{\Gamma(q+1)}\|B\|\left\|\mathbb{W}^{-1}\right\|\right] \psi\left(\mathcal{B}_{K}\right)
\end{aligned}
$$

Thus,

$$
\begin{aligned}
\psi\left(\mathcal{P}\left(\mathcal{B}_{K}(t)\right)\right) & \leq \chi\left(\Theta\left(\mathcal{B}_{K}(t)\right)\right) \\
& \leq \frac{a^{q} M_{1}}{\Gamma(q+1)} L\left[1+\frac{a^{q} M_{1}}{\Gamma(q+1)}\|B\|\left\|\mathbb{W}^{-1}\right\|\right] \psi\left(\mathcal{B}_{K}\right)
\end{aligned}
$$

which implies $\psi\left(\mathcal{B}_{K}\right)=0$ due to the condition (14) and $\nu\left(\mathcal{P}\left(\mathcal{B}_{K}\right)\right) \geq \nu\left(\mathcal{B}_{K}\right)$. The proof is complete.

Lemma 3.5. If $\nu\left(\mathcal{P}\left(\mathcal{B}_{K}\right)\right) \geq \nu\left(\mathcal{B}_{K}\right)$ then $\bmod _{c}\left(\mathcal{B}_{K}\right)=0$. 
Proof. To achieve our aim, we need to prove $\mathcal{P}\left(\mathcal{B}_{K}\right)$ is equicontinuous. Let $x \in \mathcal{B}_{K}$ and $t^{\prime}, t^{\prime \prime} \in J$ such that $0<t^{\prime}<t^{\prime \prime}$, then

$$
\begin{aligned}
& \left\|(\mathcal{P} x)\left(t^{\prime \prime}\right)-(\mathcal{P} x)\left(t^{\prime}\right)\right\| \\
& \leq\left\|\mathscr{T}_{(A, E)}\left(t^{\prime \prime}\right) x_{0}-\mathscr{T}_{(A, E)}\left(t^{\prime}\right) x_{0}\right\| \\
& +\| \int_{0}^{t^{\prime \prime}}\left(t^{\prime \prime}-s\right)^{q-1} \mathscr{S}_{(A, E)}\left(t^{\prime \prime}-s\right) f(s, x(s)) d s \\
& -\int_{0}^{t^{\prime}}\left(t^{\prime}-s\right)^{q-1} \mathscr{S}_{(A, E)}\left(t^{\prime}-s\right) f(s, x(s)) d s \| \\
& +\| \int_{0}^{t^{\prime \prime}}\left(t^{\prime \prime}-s\right)^{q-1} \mathscr{S}_{(A, E)}\left(t^{\prime \prime}-s\right) B u(s) d s \\
& -\int_{0}^{t^{\prime}}\left(t^{\prime}-s\right)^{q-1} \mathscr{S}_{(A, E)}\left(t^{\prime}-s\right) B u(s) d s \| \\
& \leq\left\|\mathscr{T}_{(A, E)}\left(t^{\prime \prime}\right)-\mathscr{T}_{(A, E)}\left(t^{\prime}\right)\right\|\left\|x_{0}\right\| \\
& +\int_{0}^{t^{\prime \prime}}\left|\left(t^{\prime \prime}-s\right)^{q-1}-\left(t^{\prime}-s\right)^{q-1}\right|\left\|\mathscr{S}_{(A, E)}\left(t^{\prime \prime}-s\right) f(s, x(s))\right\| d s \\
& +\int_{0}^{t^{\prime}}\left(t^{\prime}-s\right)^{q-1}\left\|\left[\mathscr{S}_{(A, E)}\left(t^{\prime \prime}-s\right)-\mathscr{S}_{(A, E)}\left(t^{\prime}-s\right)\right] f(s, x(s))\right\| d s \\
& +\int_{0}^{t^{\prime \prime}}\left|\left(t^{\prime \prime}-s\right)^{q-1}-\left(t^{\prime}-s\right)^{q-1}\right|\left\|\mathscr{S}_{(A, E)}\left(t^{\prime \prime}-s\right) B u(s)\right\| d s \\
& +\int_{0}^{t^{\prime}}\left(t^{\prime}-s\right)^{q-1}\left\|\left[\mathscr{S}_{(A, E)}\left(t^{\prime \prime}-s\right)-\mathscr{S}_{(A, E)}\left(t^{\prime}-s\right)\right] B u(s)\right\| d s \\
& +\int_{t^{\prime}}^{t^{\prime \prime}}\left(t^{\prime}-s\right)^{q-1}\left\|\mathscr{S}_{(A, E)}\left(t^{\prime \prime}-s\right) f(s, x(s))\right\| d s \\
& +\int_{t^{\prime}}^{t^{\prime \prime}}\left(t^{\prime}-s\right)^{q-1}\left\|\mathscr{S}_{(A, E)}\left(t^{\prime \prime}-s\right) B u(s)\right\| d s \\
& \leq I_{1}+I_{2}+I_{3}+I_{4}+I_{5}+I_{6}+I_{7},
\end{aligned}
$$

where

$$
\begin{aligned}
I_{1} & =\left\|\mathscr{T}_{(A, E)}\left(t^{\prime \prime}\right)-\mathscr{T}_{(A, E)}\left(t^{\prime}\right)\right\|\left\|x_{0}\right\| \\
I_{2} & =\frac{M_{1}}{\Gamma(q)} \int_{0}^{t^{\prime \prime}}\left[\left(t^{\prime}-s\right)^{q-1}-\left(t^{\prime \prime}-s\right)^{q-1}\right] g_{K}(s) d s \\
I_{3} & =\sup _{s \in\left[0, t^{\prime}\right]}\left\|\mathscr{S}_{(A, E)}\left(t^{\prime \prime}-s\right)-\mathscr{S}_{(A, E)}\left(t^{\prime}-s\right)\right\| \int_{0}^{t^{\prime}}\left(t^{\prime}-s\right)^{q-1} g_{K}(s) d s,
\end{aligned}
$$




$$
\begin{aligned}
I_{4} & =\frac{M_{1}\|B\|}{\Gamma(q)} \int_{0}^{t^{\prime \prime}}\left[\left(t^{\prime}-s\right)^{q-1}-\left(t^{\prime \prime}-s\right)^{q-1}\right]\|u(s)\| d s \\
I_{5} & =\sup _{s \in\left[0, t^{\prime}\right]}\left\|\mathscr{S}_{(A, E)}\left(t^{\prime \prime}-s\right)-\mathscr{S}_{(A, E)}\left(t^{\prime}-s\right)\right\|\|B\| \int_{0}^{t^{\prime}}\left(t^{\prime}-s\right)^{q-1}\|u(s)\| d s \\
I_{6} & :=\frac{M_{1}}{\Gamma(q)} \int_{t^{\prime}}^{t^{\prime \prime}}\left(t^{\prime}-s\right)^{q-1} g_{K}(s) d s \\
I_{7} & :=\frac{M_{1}\|B\|}{\Gamma(q)} \int_{t^{\prime}}^{t^{\prime \prime}}\left(t^{\prime}-s\right)^{q-1}\|u(s)\| d s
\end{aligned}
$$

Note that Lemma 2.9(ii), $\mathscr{T}_{(A, E)}(t)$ and $\mathscr{S}_{(A, E)}(t)$ are continuous in the uniform operator topology for $t \geq 0, \sup _{s \in J}\left|g_{K}(s)\right|<\infty$ and $u(\cdot)$ is bounded by (13). We can obtain the terms $I_{1}, I_{3}, I_{5}, I_{6}, I_{7} \rightarrow 0$ as $t^{\prime \prime} \rightarrow t^{\prime}$. Moreover, applying

$$
\int_{0}^{t^{\prime \prime}}\left[\left(t^{\prime}-s\right)^{q-1}-\left(t^{\prime \prime}-s\right)^{q-1}\right] d s=\frac{t^{\prime q}-t^{\prime \prime q}+\left(t^{\prime \prime}-t^{\prime}\right)^{q}}{q}
$$

one can check the terms $I_{2}, I_{4} \rightarrow 0$ as $t^{\prime \prime} \rightarrow t^{\prime}$. Thus, $\mathcal{P}\left(\mathcal{B}_{K}\right)$ is equicontinuous.

Hence, $\bmod _{c}\left(\mathcal{P}\left(\mathcal{B}_{K}\right)\right)=0$. This implies that $\bmod _{c}\left(\mathcal{B}_{K}\right)=0$ from $\nu\left(\mathcal{P}\left(\mathcal{B}_{K}\right)\right) \geq$ $\nu\left(\mathcal{B}_{K}\right)$. The proof is complete.

Lemma 3.6. The operator $\mathcal{P}$ defined by (11) is $\nu$-condensing on $\mathcal{B}_{K}$.

Proof. It follows from Lemmas 3.4 and 3.5 that $\nu\left(\mathcal{B}_{K}\right)=(0,0)$. The regularity property of $v$ implies the relative compactness of $\mathcal{B}_{K}$. It follows from Definition 2.3 that $\mathcal{P}$ is $\nu$-condensing on $\mathcal{B}_{K}$.

For $\hat{\lambda} \in(0,1]$, consider a one-parameter family of maps $\mathcal{H}:[0,1] \times C(J, X) \rightarrow$ $C(J, X)$ given by

$$
(\hat{\lambda}, x) \rightarrow \mathcal{H}(\hat{\lambda}, x)=\hat{\lambda} \mathcal{P}(x)
$$

Lemma 3.7. The fixed point set of the family of maps $\mathcal{H}$ :

$$
\text { Fix } \mathcal{H}=\{x \in \mathcal{H}(\hat{\lambda}, x) \text { for some } \hat{\lambda} \in(0,1]\}
$$

has a priori bounded.

Proof. The result can be derived by Lemma 3.2 immediately. We omit it here.

Now we are ready to state the main results in this paper.

Theorem 3.8. Assume $\left[H_{1}\right]-\left[H_{5}\right]$ are satisfied. Then the system (1) is controllable on $J$ provided that the conditions (12) and (14) hold.

Proof. To obtain our conclusion, we need to prove $\mathcal{P}$ has a fixed point in $\mathcal{B}_{K}$. In fact, it follows from Lemmas 3.2 and 3.6 that $\mathcal{P}: \mathcal{B}_{K} \rightarrow \mathcal{B}_{K}$ is $\nu$-condensing map. By Theorem 2.5, $\mathcal{P}$ has a fixed point in $\mathcal{B}_{K}$. This implies that any fixed point of $\mathcal{P}$ is just a mild solution of the system $(1)$ on $J$ which satisfying $(\mathcal{P} x)(a)=x_{1}$ with $u(t)$ given by (10). Therefore, the system (1) is controllable on $J$.

COROLlary 3.9. Let the assumptions of Theorem 3.8 be satisfied. The set of mild solutions of the system (1) is a nonempty and compact subset of $C(J, X)$ with $u(t)$ given by (10).

Proof. It follows from Lemma 3.7 that we can take a closed ball $\mathcal{B}_{K}$ to contain the set Fix $\mathcal{H}$ inside itself. Moreover, $\mathcal{P}$ maps $\mathcal{B}_{K}$ into $C(J, X)$ and is $\nu$-condensing map. By Theorem 2.5, we have the conclusion. 


\section{Example}

Take $X=U=L^{2}[0, \pi]$. We consider the following fractional partial differential equation with control

$$
\left\{\begin{array}{l}
{ }_{0}^{C} D_{t}^{\frac{4}{5}}\left(x(t, y)-x_{y y}(t, y)\right)=x_{y y}(t, y)+\mu t^{2}\left(\sin \frac{x(t, y)}{t}-\sin _{y y} \frac{x(t, y)}{t}\right)+B u(t), \\
\left(155 \in[0, \pi], t \in J_{1}=[0,1], 0<\mu<\infty\right. \\
x(t, 0)=x(t, \pi)=0, t \geq 0 \\
x(0, y)-x_{y y}(0, y)=x_{0}(y), 0 \leq y \leq \pi
\end{array}\right.
$$

Define $A: D(A) \subset X \rightarrow X$ by $A x=x_{y y}$ and $E: D(E) \subset X \rightarrow X$ by $E x=$ $x-x_{y y}$ respectively, where each domain $D(A), D(E)$ is given by $\left\{x \in X: x, x_{y}\right.$ are absolutely continuous, $\left.x_{y y} \in X, x(0)=x(\pi)=0\right\}$.

It follows from Theorem 2.2 in $[\mathbf{1}]$ that the pair $(A, E)$ can generate a propagation family $\{W(t), t \geq 0\}$ of uniformly bounded and $\{W(t), t \geq 0\}$ is norm continuous for $t>0$ and $\|W(t)\| \leq 1$. Meanwhile, it follows from [39] that $A$ and $E$ can be written as $A x=-\sum_{n=1}^{\infty} n^{2}\left\langle x, x_{n}\right\rangle, x \in D(A)$ and $E x=\sum_{n=1}^{\infty}(1+$ $\left.n^{2}\right)\left\langle x, x_{n}\right\rangle x_{n}, x \in D(E)$, respectively, where $x_{n}(y)=\sqrt{\frac{2}{\pi}} \sin n y, n=1,2, \cdots$ is the orthonormal set of eigenfunctions of $A$. Hence for any $x \in D(E), \lambda>0$ we obtain

$$
(\lambda E-A)^{-1} E x=\sum_{n=1}^{\infty} \frac{1+n^{2}}{\lambda\left(1+n^{2}\right)+n^{2}}\left\langle x, x_{n}\right\rangle x_{n}=\sum_{n=1}^{\infty} \int_{0}^{\infty} e^{-\lambda t} e^{-\frac{n^{2}}{1+n^{2}} t} d t\left\langle x, x_{n}\right\rangle x_{n} .
$$

Therefore, $\{W(t), t \geq 0\}$ can be generated by $-A E^{-1}$ and written as

$$
W(t) x:=\sum_{n=1}^{\infty} e^{-\frac{n^{2}}{1+n^{2}} t}\left\langle x, x_{n}\right\rangle x_{n}
$$

Then, $\mathscr{T}_{(A, E)}(\cdot)$ and $\mathscr{S}_{(A, E)}(\cdot)$ can be written as

$$
\begin{aligned}
& \mathscr{T}_{(A, E)}(t) x=\int_{0}^{\infty} \xi_{\frac{4}{5}}(\theta) \sum_{n=1}^{\infty} e^{-\frac{n^{2}}{1+n^{2}} t^{\frac{4}{5} \theta}}\left\langle x, x_{n}\right\rangle x_{n} d \theta, \\
& \mathscr{S}_{(A, E)}(t) x=\frac{4}{5} \int_{0}^{\infty} \theta \xi_{\frac{4}{5}}(\theta) \sum_{n=1}^{\infty} e^{-\frac{n^{2}}{1+n^{2}} t^{\frac{4}{5}} \theta}\left\langle x, x_{n}\right\rangle x_{n} d \theta .
\end{aligned}
$$

Clearly, $\left\|\mathscr{T}_{(A, E)}(t)\right\| \leq 1$ and $\left\|\mathscr{S}_{(A, E)}(t)\right\| \leq \frac{1}{\Gamma\left(\frac{4}{5}\right)}$ for $t \geq 0$.

Next, $B: U \rightarrow D(E)$ is defined by $B=b I, b>0$ and defined by

$$
\mathbb{W} u=b \int_{0}^{1}(1-s)^{-\frac{1}{5}} \mathscr{S}_{(A, E)}(1-s) u(s, y) d s
$$


Since $q=\frac{4}{5}>\frac{1}{2}$, we can take $p=2$ and $\mathscr{U}=L^{2}\left(J_{1}, U\right)$ and so $K_{\frac{4}{5}}=\sqrt{\frac{5}{3}}$. It is easy to show that $\mathbb{W}$ is surjective. Indeed, if $u(s, y):=x(y) \in \mathscr{U}$. Then

$$
\begin{aligned}
\mathbb{W} u & =b \int_{0}^{1}(1-s)^{-\frac{1}{5}} \frac{4}{5} \int_{0}^{\infty} \theta \xi_{\frac{4}{5}}(\theta) \sum_{n=1}^{\infty} e^{-\frac{n^{2}}{1+n^{2}}(1-s)^{\frac{4}{5}} \theta}\left\langle x, x_{n}\right\rangle x_{n} d \theta d s \\
& =b \int_{0}^{\infty} \xi_{\frac{4}{5}}(\theta) \sum_{n=1}^{\infty} \int_{0}^{1} \frac{4}{5} \theta(1-s)^{-\frac{1}{5}} e^{-\frac{n^{2}}{1+n^{2}}(1-s)^{\frac{4}{5}} \theta} d s\left\langle x, x_{n}\right\rangle x_{n} d \theta \\
& =b \int_{0}^{\infty} \xi_{\frac{4}{5}}(\theta) \sum_{n=1}^{\infty} \int_{0}^{1} \frac{1+n^{2}}{n^{2}} \frac{d}{d s}\left[e^{-\frac{n^{2}}{1+n^{2}}(1-s)^{\frac{4}{5}} \theta}\right] d s\left\langle x, x_{n}\right\rangle x_{n} d \theta \\
& =b \int_{0}^{\infty} \xi_{\frac{4}{5}}(\theta) \sum_{n=1}^{\infty} \frac{1+n^{2}}{n^{2}}\left[1-e^{-\frac{n^{2}}{1+n^{2}} \theta}\right]\left\langle x, x_{n}\right\rangle x_{n} d \theta \\
& =b \sum_{n=1}^{\infty} \frac{1+n^{2}}{n^{2}}\left[1-\mathbb{E}_{\frac{4}{5}}\left(-\frac{n^{2}}{1+n^{2}}\right)\right]\left\langle x, x_{n}\right\rangle x_{n},
\end{aligned}
$$

where $\mathbb{E}_{\frac{4}{5}}$ is a Mittag-Leffler function $[\mathbf{4 0}, \mathbf{4 1}]$. So we can define a right inverse $\mathbb{W}^{-1}: X \rightarrow \mathscr{U}$ by

$$
\left(\mathbb{W}^{-1} x\right)(t, y):=\frac{1}{b} \sum_{n=1}^{\infty} \frac{n^{2}}{1+n^{2}} \frac{\left\langle x, x_{n}\right\rangle x_{n}}{1-\mathbb{E}_{\frac{4}{5}}\left(-\frac{n^{2}}{1+n^{2}}\right)}, \text { for } x=\sum_{n=1}^{\infty}\left\langle x, x_{n}\right\rangle x_{n},
$$

with

$$
\left\|\mathbb{W}^{-1}\right\|=\frac{1}{b\left(1-\mathbb{E}_{\frac{4}{5}}\left(-\frac{n^{2}}{1+n^{2}}\right)\right)} \leq \frac{1}{b\left(1-\mathbb{E}_{\frac{4}{5}}\left(-\frac{1}{2}\right)\right)} .
$$

Now $f: J_{1} \times \mathbb{R} \rightarrow \mathbb{R}$ is defined by $f(t, x(t, y))=\mu t^{2} \sin \frac{x(t, y)}{t}$. It is easy to see that $f$ is measurable for the first variable and $f(t, x)$ is continuous for the second variable. Moreover, clearly $\lim \sup _{k \rightarrow \infty} \frac{1}{k} \sup _{t \in J_{1},|x| \leq k}|f(t, x)|=0$ and $\chi\left(f\left(t, D_{1}\right)\right) \leq \mu t \chi\left(D_{1}\right) \leq \mu \chi\left(D_{1}\right)$ for any bounded set $D_{1} \subset X$ and $t \in J_{1}$. Hence $\gamma=0$ and $L=\mu$.

Define $F: J_{1} \times C(J, X) \rightarrow D(E)$ by $F(t, z)(y)=f(t, z(y))$. Now, the system (15) can be abstracted as

$$
\left\{\begin{array}{l}
{ }_{0}^{C} D_{t}^{\frac{4}{5}}(E x(t))=-A x(t)+E F(t, x(t))+E B u(t), t \in J_{1}, \\
E x(0)=E x_{0} .
\end{array}\right.
$$

From the above discussion, all the assumptions in Theorem 3.8 are satisfied, since by $\gamma=0,(12)$ holds, while (14) holds when:

$$
\frac{\mu}{\Gamma\left(\frac{9}{5}\right)}\left[1+\frac{1}{\Gamma\left(\frac{9}{5}\right)\left(1-\mathbb{E}_{\frac{4}{5}}\left(-\frac{1}{2}\right)\right)}\right]<1 .
$$

Then the system (15) is controllable on $J_{1}$.

Finally, one can numerically find that $\mu<0.229071$. It is key to compute $\mathbb{E}_{\frac{4}{5}}\left(-\frac{1}{2}\right)$. 
We only provide a possible way to compute $\mathbb{E}_{\frac{4}{5}}\left(-\frac{1}{2}\right)$. In fact, we can use the definition

$$
\mathbb{E}_{\frac{4}{5}}\left(-\frac{1}{2}\right)=\sum_{k=0}^{\infty} \frac{(-1)^{k}}{2^{k} \Gamma\left(1+\frac{5 i}{4}\right)}=\sum_{k=0}^{25} \frac{(-1)^{k}}{2^{k} \Gamma\left(1+\frac{5 i}{4}\right)}+\sum_{k=26}^{\infty} \frac{(-1)^{k}}{2^{k} \Gamma\left(1+\frac{5 i}{4}\right)} .
$$

Using Mathematica we get

$$
\sum_{k=0}^{25} \frac{(-1)^{k}}{2^{k} \Gamma\left(1+\frac{5 i}{4}\right)} \doteq 0.626879
$$

On the other hand, it holds

$$
\sum_{k=26}^{\infty} \frac{(-1)^{k}}{2^{k} \Gamma\left(1+\frac{5 i}{4}\right)} \leq \sum_{k=26}^{\infty} \frac{1}{2^{k}}=\frac{1}{2^{25}} \doteq 2.98023 \times 10^{-8} .
$$

Hence $\mathbb{E}_{\frac{4}{5}}\left(-\frac{1}{2}\right) \doteq 0.626879$. The rest computations to estimate $\mu$ is given again by Mathematica, since $\Gamma$ is built in Mathematica.

We also remark that one can compute $\mathbb{E}_{\frac{4}{5}}\left(-\frac{1}{2}\right)$ by using the formula $(21)$ in $[41]$.

\section{References}

[1] J. Liang, T. J. Xiao, Abstract degenerate Cauchy problems in locally convex spaces, J. Math. Anal. Appl., 259(2001), 398-412.

[2] K. Diethelm, The analysis of fractional differential equations, Lecture Notes in Mathematics, 2010.

[3] A. A. Kilbas, H. M. Srivastava, J. J. Trujillo, Theory and applications of fractional differential equations, in: North-Holland Mathematics Studies, vol. 204, Elsevier Science B.V., Amsterdam, 2006.

[4] K. S. Miller, B. Ross, An introduction to the fractional calculus and differential equations, John Wiley, New York, 1993.

[5] I. Podlubny, Fractional differential equations, Academic Press, San Diego, 1999.

[6] V. E. Tarasov, Fractional dynamics: Application of fractional calculus to dynamics of particles, fields and media, Springer, HEP, 2010.

[7] M. M. El-Borai, Some probability densities and fundamental solutions of fractional evolution equations, Chaos, Solitons and Fractals, 14(2002), 433-440.

[8] M. M. El-Borai, The fundamental solutions for fractional evolution equations of parabolic type, J. Appl. Math. Stochastic Anal., 3(2004), 197-211.

[9] K. Balachandran, J. Y. Park, Controllability of fractional integrodifferential systems in Banach spaces, Nonlinear Anal.:TMA, 3(2009), 363-367.

[10] K. Balachandran, S. Kiruthika, J.J. Trujillo, On fractional impulsive equations of Sobolev type with nonlocal condition in Banach spaces, Comput. Math. Appl., 62(2011), 1157-1165.

[11] K. Balachandran, Y. Zhou, J. Kokila, Relative controllability of fractional dynamical systems with delays in control, Commun. Nonlinear Sci. Numer. Simulat., 17(2012), 3508-3520.

[12] Y. Zhou, F. Jiao, Existence of mild solutions for fractional neutral evolution equations, Comput. Math. Appl., 59(2010), 1063-1077.

[13] Y. Zhou, F. Jiao, Nonlocal Cauchy problem for fractional evolution equations, Nonlinear Anal., 11(2010), 4465-4475.

[14] E. Hernández, D. O'Regan, K. Balachandran, On recent developments in the theory of abstract differential equations with fractional derivatives, Nonlinear Anal.:TMA, 73(2010), 3462-3471.

[15] E. Hernández, D. O’Regan, K. Balachandran, Existence results for abstract fractional differential equations with nonlocal conditions via resolvent operators, Indagationes Math., (2012), doi:10.1016/j.indag.2012.06.007.

[16] J. Wang, Y. Zhou, A class of fractional evolution equations and optimal controls, Nonlinear Anal., 12(2011), 262-272. 
[17] J. Wang, Y. Zhou, Existence and controllability results for fractional semilinear differential inclusions, Nonlinear Anal.:RWA, 12(2011), 3642-3653.

[18] J. Wang, Y. Zhou, Analysis of nonlinear fractional control systems in Banach spaces, Nonlinear Anal.:TMA, 74(2011), 5929-5942.

[19] J. Wang, M. Fečkan, Y. Zhou, Relaxed controls for nonlinear fractional impulsive evolution equations, J. Optim. Theory Appl., 156(2013), 13-32.

[20] R. Sakthivel, Y. Ren, N. I. Mahmudov, On the approximate controllability of semilinear fractional differential systems, Comput. Math. Appl., 62(2011), 1451-1459.

[21] R. Sakthivela, N. I. Mahmudov, J. J. Nieto, Controllability for a class of fractional-order neutral evolution control systems, Appl. Math. Comput., 218(2012), 10334-10340.

[22] R. Sakthivela, S. Suganya, S. M. Anthoni, Approximate controllability of fractional stochastic evolution equations, Comput. Math. Appl., 63(2012), 660-668.

[23] A. Debbouchea, D. Baleanu, Controllability of fractional evolution nonlocal impulsive quasilinear delay integro-differential systems, Comput. Math. Appl., 62(2011), 1442-1450.

[24] J. Wang, Y. Zhou, M. Medved', On the solvability and optimal controls of fractional integrodifferential evolution systems with infinite delay, J. Optim. Theory Appl., 152(2012), 31-50.

[25] J. Wang, Y. Zhou, W. Wei, Optimal feedback control for semilinear fractional evolution equations in Banach spaces, Syst. Control Lett., 61(2012), 472-476.

[26] J. Wang, Z. Fan, Y. Zhou, Nonlocal controllability of semilinear dynamic systems with fractional derivative in Banach spaces, J. Optim. Theory Appl., 154(2012), 292-302.

[27] J. Wang, Y. Zhou, Complete controllability of fractional evolution systems, Commun. Nonlinear Sci. Numer. Simulat., 17(2012), 4346-4355.

[28] J. Wang, Y. Zhou, W. Wei, Fractional Schrödinger equations with potential and optimal controls, Nonlinear Anal.:RWA, 13(2012), 2755-2766.

[29] R. N. Wang, D. H. Chen, T. J. Xiao, Abstract fractional Cauchy problems with almost sectorial operators, J. Differential Equations, 252(2012), 202-235.

[30] S. Kumar, N. Sukavanam, Approximate controllability of fractional order semilinear systems with bounded delay, J. Differential Equations, 252(2012), 6163-6174.

[31] K. Li, J. Peng, J. Jia, Cauchy problems for fractional differential equations with RiemannLiouville fractional derivatives, J. Funct. Anal., 263(2012), 476-510.

[32] K. Balachandran, J. P. Dauer, Controllability of functional differential systems of Sobolev type in Banach spaces, Kybernetika, 34(1998), 349-357.

[33] S. Agarwal, D. Bahuguna, Existence of solutions to Sobolev-type partial neutral differential equations, J. Appl. Math. Stoch. Anal., 2006(2006), Art. ID 16308, 10 pp.

[34] F. Li, J. Liang, H. K. Xu, Existence of mild solutions for fractional integrodifferential equations of Sobolev type with nonlocal conditions, J. Math. Anal. Appl., 391(2012), 510-525.

[35] M. Fečkan, J. Wang, Y. Zhou, Controllability of fractional functional evolution equations of Sobolev type via characteristic solution operators, J. Optim. Theory Appl., 156(2013), 7995.

[36] R. R. Akhmerov, M. I. Kamenskii, A. S. Potapov, A. E. Rodkina, B. N. Sadovskii, Measures of noncompactness and condensing operators, Birkhäser, Boston, Basel, Berlin, 1992.

[37] J. Banas̀, K. Goebel, Measure of noncompactness in Banach spaces, Lecture Notes in Pure and Appl. Math., 60, Marcel Dekker, New York, 1980.

[38] M. Kamenskii, V. Obukhovskii, P. Zecca, Condensing multivalued maps and semilinear differential inclusions in Banach spaces, de Gruyter Ser. Nonlinear Anal. Appl., vol. 7, Walter de Gruyter, Berlin, New York, 2001.

[39] J. H. Lightbourne, S. M. Rankin, A partial functional differential equation of Sobolev type, J. Math. Anal. Appl., 93(1983), 328-337.

[40] M. N. Berberan-Santos, Relation between the inverse Laplace transforms of $I\left(t^{\beta}\right)$ and $I(t)$ : Application to the Mittag-Leffler and asymptotic inverse power law relaxation functions, J. Math. Chem., 38(2005), 265-270.

[41] C. Atkinson, A. Osseiran, Rational solutions for the time-fractional diffusion equation, SIAM J. Appl. Math., 71(2011), 92-106. 
School of Mathematics and Computer Science, Guizhou Normal College, Guiyang, Guizhou 550018, P.R. China. Department of Mathematics, Guizhou University, Guiyang, Guizhou 550025, P.R. China

E-mail address: sci.jrwang@gzu.edu.cn

Department of Mathematical Analysis and Numerical Mathematics, Faculty of Mathematics, Physics and Informatics, Comenius University, Mlynská dolina, 84248 Bratislava, Slovakia, and Mathematical Institute, Slovak Academy of Sciences, Štefánikova 49, 81473 Bratislava, Slovakia

E-mail address: Michal.Feckan@fmph.uniba.sk CHINA

Department of Mathematics, Xiangtan University, Xiangtan, Hunan 411105, P.R.

E-mail address: yzhou@xtu.edu.cn 